\title{
CONSTRUCTION OF SOME NEW HADAMARD MATRICES
}

\section{Dragomir Z̆. ĐoKović}

We prove that there exist skew type Hadamard matrices of order $4 \boldsymbol{n}$ for $n=67,113$, $127,157,163,181$ and 241 which have not been constructed so far. In particular there exists a Hadamard matrix of order $4 \cdot 163$, which was unknown until now. We mention that very recently we have constructed skew type Hadamard matrices of orders $4 n$ for $n=37$ and 43 .

1.

A Hadamard matrix of order $m$ is a $(1,-1)$-matrix $H$ of order $m$ satisfying $H H^{T}=m I_{m} \cdot\left(X^{T}\right.$ denotes the transpose of a matrix $X$, and $I_{m}$ the identity matrix of order $m$.) The order $m$ of a Hadamard matrix $H$ must be 1, 2 or a multiple of 4, $m=4 n$. A $(1,-1)$-matrix $A$ of order $m$ is said to be of skew type if $A+A^{T}=2 I_{m}$. A skew Hadamard matrix is a Hadamard matrix of skew type.

It has been conjectured that Hadamard matrices as well as skew Hadamard matrices exist for all orders $m$ which are multiples of 4 . According to Appendix $K$ of the book [2, p.416] published in 1979, Hadamard matrices of order $m=4 n$ with $n<200$ were not known only for the following nine values of $\boldsymbol{n}$ :

$$
67,103,107,127,151,163,167,179,191 .
$$

For orders of skew Hadamard matrices $m=4 n$ with $n$ odd and $n<250$ only the following 53 values were dubious (see [5]) :

$$
\begin{gathered}
29,37,39,43,47,49,59,65,67,69,81,89,93,97,101,103,107,109,113,119,121, \\
127,129,133,145,149,151,153,157,163,167,169,177,179,181,191,193, \\
201,205,209,213,217,219,223,225,229,233,235,239,241,245,247,249 .
\end{gathered}
$$

The number 67 has been removed from the list (1) by Sawade [3], and the numbers 103, 127 and 151 by Yamada [7, Theorem 4]. From the list (2), the number 29 has been removed by Szekeres [6] and the numbers 37 and 43 were removed recently by the author [1]. Here we announce the existence of skew Hadamard matrices of orders $m=4 n$ for $n=67,113,127,157,163,181$ and 241 . Hence these numbers should be removed from the list (2) and consequently the number 163 should be removed from the list (1).

\section{Received 22 April 1991}

This work was supported by the NSERC of Canada Grant A-5285.

Copyright Clearance Centre, Inc. Serial-fee code: 0004-9729/92 \$A2.00+0.00. 
2.

We shall identify the integer $i \in\{0,1,2, \ldots, n-1\}$ with the corresponding residue class modulo $n$. We say that subsets $S_{i}, 1 \leqslant i \leqslant k$, of $\{0,1,2, \ldots, n-1\}$ are

$$
k-\left(n ; n_{1}, \ldots, n_{k} ; \lambda\right)
$$

supplementary difference sets modulo $n$ (abbreviated as sds) if $\left|S_{i}\right|=n_{i}$ for $1 \leqslant i \leqslant k$ and for each non-zero residue $r$ modulo $n$ the congruences

$$
x_{i}-y_{i} \equiv r(\bmod n)
$$

have in total exactly $\lambda$ solutions with $x_{i}, y_{i} \in S_{i}$ and $1 \leqslant i \leqslant k$ ( $i$ is not fixed). Our construction of skew Hadamard matrices is based on some new $4-\left(n ; n_{1}, \ldots, n_{4} ; \lambda\right)$ sds with $\lambda=n_{1}+\cdots+n_{4}-n$.

We say that a subset $S$ of $\{1,2, \ldots, n-1\}$ is of skew type if

$$
i \in S \quad \Longleftrightarrow \quad-i \notin S \text {. }
$$

They exist if and only if $n$ is odd. Given any subset $S$ of $\{1,2, \ldots, n-1\}$ we denote by $A_{S}=\left(a_{i j}\right)$ the circulant $(1,-1)$-matrix of order $n, 0 \leqslant i, j \leqslant n-1$, whose first row is given by

$$
a_{0, j}= \begin{cases}-1 & \text { if } j \in S, \\ 1 & \text { if } j \notin S .\end{cases}
$$

Since $0 \notin S$, all diagonal entries of $A_{S}$ are 1's. $A_{S}$ is of skew type if and only if $S$ is of skew type. For later use we introduce the permutation matrix $R=\left(r_{i j}\right)$ of order $n$, $0 \leqslant i, j \leqslant n-1$, such that

$$
r_{i j}= \begin{cases}1 & \text { if } i+j \equiv-1(\bmod n) \\ 0 & \text { otherwise }\end{cases}
$$

3.

We say that a $(1,-1)$-matrix $H$ of order $m=4 n$ is of Goethals-Seidel type if

$$
H=\left(\begin{array}{cccc}
A_{1} & A_{2} R & A_{3} R & A_{4} R \\
-A_{2} R & A_{1} & -A_{4}^{T} R & A_{3}^{T} R \\
-A_{3} R & A_{4}^{T} R & A_{1} & -A_{2}^{T} R \\
-A_{4} R & -A_{3}^{T} R & A_{2}^{T} R & A_{1}
\end{array}\right)
$$

where $A_{i}$ are circulant matrices of order $n$ and $R$ is the matrix defined in the previous section. Such $B$ is a Hadamard matrix if and only if

$$
\sum_{i=1}^{4} A_{i} A_{i}^{T}=4 n I_{n}
$$


see [4]. Furthermore $H$ is of skew type if and only if $A_{1}$ is of skew type. All Hadamard matrices constructed in this paper are of Goethals-Seidel type.

Let $S=\left(S_{1}, S_{2}, S_{3}, S_{4}\right)$ be a 4 -tuple of subsets of $\{1,2, \ldots, n-1\}$ and let $H_{S}$ be the matrix $H$ above with $A_{i}=A_{S_{i}}, 1 \leqslant i \leqslant 4$. We write $n_{i}$ for the cardinality $\left|S_{i}\right|$ of $S_{i}$. The following proposition is well known.

PROPOSITION 1. (See [4]) The matrix $H_{S}$ is a Hadamard matrix if and only if $S_{1}, S_{2}, S_{3}, S_{4}$ are $4-\left(n ; n_{1}, n_{2}, n_{3}, n_{4} ; \sum_{i=1}^{4} n_{i}-n\right)$ supplementary difference sets modulo $n$. Furthermore $H_{S}$ is of skew type if and only if $S_{1}$ is of skew type.

4.

In all cases that we consider below, $n$ is a prime number and so $G=\{1,2, \ldots, n-1\}$ is a group under multiplication modulo $n$. We choose a subgroup $H$ of order $k$ for some odd integer $k>1$ and set $b=[G: H]=(n-1) / k$. Then we enumerate the cosets of $H$ in $G$ :

$$
\alpha_{0}, \alpha_{1}, \ldots, \alpha_{b-1}
$$

In all cases our enumeration is such that

$$
\alpha_{2 i+1}=-1 \cdot \alpha_{2 i}, \quad 0 \leqslant i<b / 2,
$$

and so it suffices to list the cosets $\alpha_{2 i}$ only. The sets $S_{i}$ are constructed as unions of cosets $\alpha_{i}$. By the above proposition, in order to construct a skew Hadamard matrix of order $m=4 n$ it suffices to produce four such sets $S_{i}$ with $S_{1}$ of skew type which are $4-\left(n ; n_{1}, \ldots, n_{4} ; \lambda\right)$ sds where $\lambda=\sum n_{i}-n$ and $n_{i}=\left|S_{i}\right|$.

THEOREM 2. There exist skew Hadamard matriçes of order $4 n$ for $n=67,113$, $127,157,163,181$ and 241.

Proof: It suffices to list the required sds's. Although in some cases we have constructed several non-equivalent sds's, for the sake of brevity, we shall give here only one sds for each $n$ listed in the theorem.

CASE. $n=67$. Let $H=\{1,29,37\}$ be the subgroup of $G$ of order 3. Enumerate the cosets $\alpha_{2 i}$ as follows :

$$
\begin{aligned}
& \alpha_{0}=H, \alpha_{2}=2 H, \alpha_{4}=3 H, \alpha_{6}=4 H, \alpha_{8}=5 H, \alpha_{10}=6 H, \\
& \alpha_{12}=8 H, \alpha_{14}=10 H, \alpha_{16}=12 H, \alpha_{18}=15 H, \alpha_{20}=17 H,
\end{aligned}
$$

and recall that $\alpha_{2 i+1}=-1 \cdot \alpha_{2 i}$. Then the sets

$$
\begin{aligned}
& S_{1}=\cup \alpha_{i}, \quad i \in\{0,3,5,6,9,10,13,14,17,18,20\}, \\
& S_{2}=\cup \alpha_{i}, \quad i \in\{0,2,4,9,11,12,13,16,19,21\}, \\
& S_{3}=\cup \alpha_{i}, \quad i \in\{1,3,6,10,11,13,14,16,20,21\} \\
& S_{4}=\cup \alpha_{i}, \quad i \in\{2,4,6,8,9,11,14,17,19\},
\end{aligned}
$$


are $4-(67 ; 33,30,30,27 ; 53)$ sds with $S_{1}$ of skew type.

CASE. $n=113$. Here $H=\{1,16,28,30,49,106,109\}$ is the subgroup of $G$ of order 7 . We enumerate the cosets $\alpha_{2 i}$ as follows :

$$
\alpha_{0}=H, \alpha_{2}=2 H, \alpha_{4}=3 H, \alpha_{6}=5 H, \alpha_{8}=6 H, \alpha_{10}=9 H, \alpha_{12}=10 H, \alpha_{14}=13 H
$$

The sets

$$
\begin{aligned}
& S_{1}=\cup \alpha_{i}, \quad i \in\{0,3,4,6,8,10,13,14\}, \\
& S_{2}=\cup \alpha_{i}, \quad i \in\{1,3,8,9,10,11,12,13\}, \\
& S_{3}=\cup \alpha_{i}, \quad i \in\{0,2,3,5,6,7,12\}, \\
& S_{4}=\cup \alpha_{i}, \quad i \in\{1,2,3,5,8,9,15\},
\end{aligned}
$$

are $4-(113 ; 56,56,49,49 ; 97)$ sds with $S_{1}$ of skew type.

CASE. $n=127$. Here $H=\{1,2,4,8,16,32,64\}$ is the subgroup of $G$ of order 7 . We enumerate the cosets $\alpha_{2 i}$ as follows :

$$
\begin{gathered}
\alpha_{0}=H, \alpha_{2}=3 H, \alpha_{4}=5 H, \alpha_{6}=7 H, \alpha_{8}=9 H, \alpha_{10}=11 H \\
\alpha_{12}=13 H, \alpha_{14}=19 H, \alpha_{16}=21 H
\end{gathered}
$$

The sets

$$
\begin{array}{ll}
S_{1}=\cup \alpha_{i}, & i \in\{0,3,5,7,8,10,12,14,16\}, \\
S_{2}=\cup \alpha_{i}, & i \in\{0,1,3,6,7,9,10,12,14,15\}, \\
S_{3}=\cup \alpha_{i}, & i \in\{0,1,3,4,5,7,8,9,15,16\}, \\
S_{4}=\cup \alpha_{i}, & i \in\{1,4,5,6,9,10,13,14,15,16\},
\end{array}
$$

are $4-(127 ; 63,70,70,70 ; 146)$ sds with $S_{1}$ of skew type. In this case $S_{1}$ is the $(127,63,31)$ difference set consisting of the non-zero quadratic residues modulo 127 and consequently $S_{2}, S_{3}, S_{4}$ are $3-(127 ; 70,70,70 ; 115)$ sds.

CASE. $n=157$. Here $H=\{1,14,16,39,46,67,75,93,99,101,108,130,153\}$ is the subgroup of $G$ of order 13 . We enumerate the cosets $\alpha_{2 i}$ as follows :

$$
\alpha_{0}=H, \alpha_{2}=2 H, \alpha_{4}=3 H, \alpha_{6}=5 H, \alpha_{8}=9 H, \alpha_{10}=15 H
$$

The sets

$$
\begin{array}{ll}
S_{1}=\cup \alpha_{i}, & i \in\{0,2,5,7,8,11\}, \\
S_{2}=\cup \alpha_{i}, & i \in\{0,4,5,6,9,11\}, \\
S_{3}=\cup \alpha_{i}, & i \in\{6,7,8,9,10,11\}, \\
S_{4}=\cup \alpha_{i}, & i \in\{0,5,6,7,8,10,11\},
\end{array}
$$

are $4-(157 ; 78,78,78,91 ; 168)$ sds with $S_{1}$ of skew type. 
CASE. $n=163$. Here $H=\{1,38,40,53,58,85,104,133,140\}$ is the subgroup of $G$ of order 9 . We enumerate the cosets $\alpha_{2 i}$ as follows :

$$
\begin{gathered}
\alpha_{0}=H, \alpha_{2}=2 H, \alpha_{4}=3 H, \alpha_{6}=5 H, \alpha_{8}=6 H, \alpha_{10}=9 H, \\
\alpha_{12}=10 H, \alpha_{14}=15 H, \alpha_{16}=18 H .
\end{gathered}
$$

The sets

$$
\begin{aligned}
& S_{1}=\cup \alpha_{i}, \quad i \in\{0,2,5,6,9,10,13,14,17\}, \\
& S_{2}=\cup \alpha_{i}, \quad i \in\{0,1,7,10,12,15,16,17\}, \\
& S_{3}=\cup \alpha_{i}, \quad i \in\{0,1,3,5,8,13,15,16,17\}, \\
& S_{4}=\cup \alpha_{i}, \quad i \in\{3,6,7,8,11,12,13,14,16,17\},
\end{aligned}
$$

are $4-(163 ; 81,72,81,90 ; 161)$ sds with $S_{1}$ of skew type.

CASE. $n=181$. Here $H=\{1,39,43,48,62,65,73,80,132\}$ is the subgroup of $G$ of order 9 . We enumerate the cosets $\alpha_{2 i}$ as follows :

$$
\begin{gathered}
\alpha_{0}=H, \alpha_{2}=2 H, \alpha_{4}=3 H, \alpha_{6}=4 H, \alpha_{8}=6 H, \alpha_{10}=7 H, \\
\alpha_{12}=8 H, \alpha_{14}=12 H, \alpha_{16}=13 H, \alpha_{18}=24 H .
\end{gathered}
$$

The sets

$$
\begin{array}{ll}
S_{1}=\cup \alpha_{i}, & i \in\{0,3,5,6,8,10,13,15,16,19\}, \\
S_{2}=\cup \alpha_{i}, & i \in\{4,5,7,8,11,14,15,16,18,19\}, \\
S_{3}=\cup \alpha_{i}, & i \in\{0,4,10,11,13,15,16,18,19\}, \\
S_{4}=\cup \alpha_{i}, & i \in\{2,4,5,7,11,13,15,17,19\},
\end{array}
$$

are $4-(181 ; 90,90,81,81 ; 161)$ sds with $S_{1}$ of skew type.

CASE. $n=241$. Here

$$
H=\{1,15,24,54,87,91,94,98,100,119,160,183,205,225,231\}
$$

is the subgroup of $G$ of order 15 . We enumerate the cosets $\alpha_{2 i}$ as follows :

$$
\begin{gathered}
\alpha_{0}=H, \alpha_{2}=2 H, \alpha_{4}=4 H, \alpha_{6}=5 H, \alpha_{8}=7 H, \alpha_{10}=13 H, \\
\alpha_{12}=19 H, \alpha_{14}=35 H .
\end{gathered}
$$

The sets

$$
\begin{array}{ll}
S_{1}=\cup \alpha_{i}, & i \in\{0,2,4,6,8,11,12,14\}, \\
S_{2}=\cup \alpha_{i}, & i \in\{1,3,4,6,7,13,14,15\}, \\
S_{3}=\cup \alpha_{i}, & i \in\{6,8,9,10,12,13,14,15\}, \\
S_{4}=\cup \alpha_{i}, & i \in\{3,4,5,9,10,13,14\},
\end{array}
$$

are $4-(241 ; 120,120,120,105 ; 224)$ sds with $S_{1}$ of skew type.

This completes the proof.

NOTE ADDED IN PROOF. If we interchange $S_{1}$ and $S_{4}$ in the sds given above for the case $n=241$, then the matrix $H$ is a Hadamard matrix of order 964 of maximal excess 29884 . 


\section{REFERENCES}

[1] D. Ż. Đoković, 'Skew Hadamard matrices of order $4 \cdot 37$ and $4 \cdot 43$ ', J. Combin. Theory Ser. $A$ (to appear).

[2] A. Geramita and J. Seberry, Orthogonal Designs (M. Dekker, New York - Basel, 1979).

[3] K. Sawade, 'A Hadamard matrix of order 268', Graphs Combin. 1 (1985), 185-187.

[4] J. Seberry Wallis, 'Hadamard matrices, Part IV', in Combinatorics : Room squares, sum free sets, Hadamard matrices 292: Lecture Notes in Mathematics, by W.D. Wallis, A. Penfold Street, J. Seberry Wallis (Springer-Verlag, Berlin, Heidelberg, New York, 1972).

[5] J. Seberry, 'On skew Hadamard matrices', Ars Combin. 6 (1978), 255-275.

[6] G. Szekeres, 'A note on skew type orthogonal \pm 1 matrices', in Combinatorics: Colloq. Math. Soc. János Bolyai, Editors A. Hajnal, L. Lovász, and V. T. Sós, pp. 489-498 (North Holland, 1988).

[7] M. Yamada, 'Some new series of Hadamard matrices', J. Austral. Math. Soc. Ser. A 46 (1989), 371-383.

Department of Pure Mathematics

University of Waterloo Waterloo, Ontario

Canada N2L 3G1 